\title{
Chewing gum benefits sustained attention in the absence of task degradation
}

Andrew J. Johnson, ${ }^{1}$ Mohammed Muneem, ${ }^{2}$ and Christopher Miles ${ }^{3}$

${ }^{1}$ Psychology Research Centre

Bournemouth University, Poole,

United Kingdom
${ }^{2}$ Department of Psychology and

Behavioural Sciences,

Coventry University,

Coventry, United Kingdom

\author{
${ }^{3}$ School of Psychology \\ Cardiff University, \\ Cardiff, \\ United Kingdom
}

$\begin{array}{ll}\text { Corresponding Author: } & \text { Andrew J. Johnson } \\ \text { Address for Correspondence: } & \text { Psychology Research Centre } \\ & \text { Bournemouth University } \\ & \text { Fern Barrow } \\ & \text { Poole } \\ & \text { BH12 5BB }\end{array}$

Email: andjohnson@bournemouth.ac.uk

Word Count:

Figures: 3551 (excluding abstract and reference section) 


\section{Acknowledgements}

The authors thank two anonymous reviewers for their comments and Anthony McGuffie for his assistance in the programming of the Sustained Attention Response Task (SART). 


\begin{abstract}
Objectives: The present study examined the effect of chewing gum on sustained attention and associated changes in subjective alertness.

Methods: In a within-participants design, 20 participants completed an extended version of the sustained attention response task (SART: Robertson et al., 1997), both with and without chewing gum. Self-rated measures of alertness, contentedness, and calmness were taken before and after the SART.

Results: Chewing gum was associated with improved attentional task performance. This finding was not contingent upon a general decrease in attentional performance and was apparent at all stages of the task. Subjective measures of alertness, contentedness, and calmness were higher following the chewing of gum. Changes in sustained attention covaried with subjective alertness

Discussion: The effects of chewing gum on attention and alertness are consistent with past literature and were not contingent on declines in attention. Additionally, we found evidence that gum-induced changes in self-rated alertness and attention are related. We found no support for the proposition that chewing gum can impair attention due to the division of resources.
\end{abstract}

Keywords: Chewing gum, Attention, Alertness, Mood 


\section{Introduction}

A number of recent studies have demonstrated that chewing gum enhances both subjective perceptions of alertness ${ }^{1-4}$ and performance on sustained attention tasks ${ }^{2,5-7}$. Two mechanisms have been proposed to account for these effects ${ }^{1}$. The first suggests that chewing gum increases cerebral blood flow to fronto-temporal regions ${ }^{8}$ and increases cerebral activity ${ }^{9,10}$. The enhanced delivery of both oxygenated blood and glucose may act to enhance cognition ${ }^{11}$. The second suggests that flavour acts to influence self-rated mood. For example, exposure to mint odour has been shown to both attenuate the rise in physiological markers of sleepiness ${ }^{12}$ and improve performance on a behavioural vigilance task ${ }^{13}$.

In respect to gum-induced improvements on attentional task performance ${ }^{2,5-7}, \mathrm{Smith}^{2}$ showed that chewing gum improved both sustained and focussed attention, and Allen \& Smith $^{6}$ demonstrated that the attentional benefit of chewing gum is acute, i.e. it did not transfer to subsequent task performance completed in the absence of gum. The absence of a transfer effect is interesting considering that peak cortical blood flow occurs 10-minutes after gum is discarded ${ }^{14}$, thereby suggesting that cerebral blood flow is not the mechanism responsible for attentional facilitation.

There exists a degree of variability in the literature with respect to effects of chewing gum on attention and self-rated alertness (these are broadly summarised in Table 1). Additionally, there are a number of uncertainties with respect to both the trajectory and conditions under which the effects of gum on alertness occur. For example, Onyper et al. ${ }^{15}$ argued that chewing gum initially impairs cognition (due to the division of resources) and only benefits performance (due to elevation of arousal) after gum has been discarded. Some support for this proposition is evident from vigilance data in which early stages of the task demonstrated significantly lengthened reaction times ${ }^{5-7}$ coupled with significantly fewer correct responses ${ }^{7}$ when chewing gum. However, this initial decrement was not found across other measures of attention ${ }^{6}$. Moreover, when benefits were observed, they were reported prior to gum being discarded $^{5-7}$, with no evidence of a post-chewing carry-over effect ${ }^{6}$. A further issue concerns the role of time on task effects, where some studies show that gum benefits attention in the latter stages of the task only ${ }^{5,7}$ However, Allen and Smith ${ }^{6}$ reported immediate improvements on sustained attention when chewing gum, suggesting that gum effects are not contingent on fatigue. Indeed, it should be noted that enhancements in self-reported alertness following the chewing of gum are not, in all cases, predicated on a post-task decline in subjective alertness $^{2-4}$. A further related discrepancy in the literature concerns the possibility that the positive effects of chewing gum on attention and alertness reflect the action of independent processes. For example, Smith ${ }^{16}$ reported a null effect for non-caffeinated gum on attention whilst reporting a significant increase in self-rated alertness. This dissociation is analogous to Johnson et al. ${ }^{17}$ who demonstrated disparate effects of chewing gum on the perceptions and biomarkers of alertness. In this study the dissociation was interpreted as gum affecting the peripheral nervous system but not the central nervous system, thereby averting conscious awareness of change. These findings indicate that the effects of chewing gum on subjective alertness and attention are, to some extent, independent. Indeed, such dissociation has precedence in the literature. For example, under conditions of sleep deprivation, Leproult et 
al. ${ }^{18}$ found no relationship between impairments in subjective alertness and objective alertness (selective and sustained attention). They also found that EEG changes correlated with subjective alertness but not objective alertness. Differential effects on self-rated alertness and attention might be explained by demand characteristics ${ }^{6,19,20}$. Alternatively, since alertness is conceptualised as a multi-dimensional system (both cognitively and neurologically $)^{21}$, it is perhaps unsurprising that subcomponent measures of this construct (perceptions of alertness and tonic alertness) do not perfectly correlate.

Table 1: A broad overview of significant gum effects on alertness and attention

\section{Subjective Alertness}

Pre-Task Effects

Yes

No

$n / a$

$n / a$

$n / a$

No

No

Yes

Yes

$n / a$

$n / a$

$n / a$
Post-Task Effects

Smith $^{23}$

Smith $^{2}$

Tucha et al. ${ }^{24}$

Tucha and Simpson ${ }^{5}$

Wilkinson et al. ${ }^{25}$
Yes

Yes

Yes

No

Yes

Yes

Yes

No

Yes

$n / a$

$n / a$

$n / a$

\begin{tabular}{|c|c|c|c|}
\hline Allen \& Smith ${ }^{6}$ & Yes & Yes & Yes \\
\hline Gray et al. ${ }^{22}$ & No & Yes & $n / a$ \\
\hline Johnson et al. $^{3}$ & $n / a$ & Yes & No \\
\hline Johnson et al. ${ }^{17}$ & $n / a$ & No & $n / a$ \\
\hline Scholey et al. ${ }^{1}$ & $n / a$ & Yes & Yes \\
\hline Sketchley-Kaye et al. ${ }^{4}$ & No & Yes & $n / a$ \\
\hline Smith $^{16}$ & No & Yes & $n / a$ \\
\hline Smith $^{23}$ & Yes & No & No \\
\hline Smith $^{2}$ & Yes & Yes & Yes \\
\hline Tucha et al. ${ }^{24}$ & $n / a$ & $n / a$ & Yes \\
\hline Tucha and Simpson ${ }^{5}$ & $n / a$ & $n / a$ & Yes \\
\hline Wilkinson et al. ${ }^{25}$ & $n / a$ & $n / a$ & Jo \\
\hline
\end{tabular}

\section{Behavioural}

Attention Effects

The present study has, therefore, three clear aims relating to both the trajectory and conditions under which chewing gum affects attention. First, participants complete a sustained attention response task (SART) ${ }^{26}$ both with and without gum, and we examine whether time on task effects are observed. The attention task is divided into three temporal blocks and we examine at which block gum-induced benefits are observed, and the extent to which this is consistent with general task-driven declines in attention. To the extent that chewing gum influences attention in a time-on-task dependent manner, an interaction is predicted between gum condition and experimental block, such that positive effects of chewing gum will be observed for the final block of the SART only. With respect to the effect of chewing gum on subjective alertness, an interaction is predicted such that chewing 
gum moderates the time on task decline in subjective alertness. Second, we examine the proposition that chewing gum initially impairs attention due to the division of resources. This will be evidenced via significantly impaired attention for the gum condition in block one of the task. Third, we examine the extent to which gum-induced changes to task performance are independent of gum-induced changes in perceived alertness. If changes in attentional responses are independent of changes in subjective alertness, then the effects of chewing gum should persist following the employment of subjective alertness as a co-variable in the analysis

\section{Method}

Participants: Twenty Coventry University Psychology undergraduates (10 male and 10 female: mean age $=21$ years 10 months) participated in exchange for course credit. G*Power software revealed that the sample size was close to the 24 required for a 0.8 probability of obtaining significance at a moderate effect size. All participants reported as free of current medications and illicit drug use. On the days of testing participants were instructed to refrain from alcohol, caffeinated products, chewing gum, and eating for up to one hour prior to testing. Ethical approval was obtained from the Coventry University Psychology Ethics Committee.

Materials: The task employed a lengthened version of the Sustained Attention Response Task $\left(\mathrm{SART}^{26}\right)$. For the purpose of analysis, this task was divided into three temporal blocks. Participants were presented with a series of 675 single-integer digits (225 in the original SART description ${ }^{26}$ ). Each block of the experiment comprised 225 digits. Each digit was presented with a $250 \mathrm{~ms}$ on-time with a $900 \mathrm{~ms}$ inter-stimulus-interval (ISI). Digits were presented in the centre of the screen using symbol font. The font size of the digits was 48, 58, $68,78,88,98$, and 108. The presentation of these font sizes was randomised throughout the sequence. Participants were instructed to respond to each digit by depressing the spacebar. The exception to this rule required participants to inhibit their response upon presentation of the digit ' 3 ', i.e. the target digit. The percentage of target digit presentations was $11 \%$ with a minimum of 6 and maximum of 10 digits intervening between target presentations.

Self-rated measures of mood were obtained from the Bond-Lader Visual Analogue Mood Scales $\left(\mathrm{VAMS}^{27}\right)$. The VAMS comprises 16 questions; for each question antonyms anchor either side of a 100mm line (e.g. alert-drowsy). The 16 questions are factored into measures for alertness, contentedness, and calmness.

In the gum condition, participants chewed a single pellet of Wrigley's Cool Breeze chewing gum throughout the SART.

Design: For the behavioural data a 2-factor (2x3) within-participants design was employed where the first factor represents gum condition (gum versus no gum) and the second factor represents SART experimental block (first, second, and third). The order of gum presentation was counterbalanced. The dependent variable was the number of correct response inhibitions following presentation of the target digit (each experimental block contained 25 target digits). In order to establish whether effects on target response inhibition reflected general response 
inhibition (i.e. task disengagement), the number of correct responses to non-targets per experimental block is also examined. Reaction times (ms) were also measured for correct responses to non-targets.

For the self-rated mood data, a 2-factor (2x2) within-participants design was employed where the first factor represents experimental condition (gum versus no-gum) and the second factor represents pre- and post-SART. The dependent variables were alertness, contentedness, and calmness.

Procedure: All participants were tested individually in a well-ventilated, quiet laboratory on two consecutive days. Prior to the SART, participants completed the Bond-Lader VAMS. Participants were then given chewing gum or not given chewing gum. Those participants in the gum condition were instructed to chew throughout the SART at their normal rate. Participants received a short practice phase (20-digits) before the SART commenced (those in the gum condition chewed throughout the practice). The task lasted approximately 13minutes, following which participants completed the Bond-Lader VAMS. Participants repeated this procedure on Day 2 in the alternate gum condition. The procedure was repeated on the second testing day.

Statistical Analysis: A 3-factor $(2 \times 2 \times 3)$ mixed ANOVA was computed on the SART performance data comparing gum and no gum conditions across the three task blocks. The between-participant variable of order of gum presentation $\left(1^{\text {st }}\right.$ versus $\left.2^{\text {nd }}\right)$ was included to check for order effects. Independent 3 -factor $(2 \times 2 \times 2)$ mixed ANOVAs were computed on the self-rated mood measures (alertness, contentedness, and calmness) comparing gum and no gum conditions before and after the SART and including the between-groups factor of gum presentation order.

\section{Results}

\section{$\underline{\text { Attentional Task Performance }}$}

Figure 1 displays the mean number of correct target response inhibitions for the gum and nogum conditions as a function of task block (0-25 targets, 26-50 targets, and 51-75 targets). A 2-factor $(2 \times 2 \times 3)$ mixed ANOVA comprising the within-participants factors of gum condition (gum and no gum) and experimental block (first, second, and final third of the SART) and between participant factor of gum presentation order (first or second) was computed on the mean number of correct target inhibition scores. The ANOVA revealed a main effect of gum condition, $F(1,18)=17.03$, $p=0.001$, partial $\eta^{2}=0.49$ (means=10.75 and 7.77 for the gum and no-gum conditions, respectively), confirming that chewing gum benefitted correct target response inhibitions across all stages of the task. Both the main effect of experimental block $\left(F(2,36)=0.44, p=0.65\right.$, partial $\left.\eta^{2}=0.02\right)$ and the chewing gum by experimental block interaction were non-significant $\left(F(2,36)=0.56, p=0.58\right.$, partial $\left.\eta^{2}=0.03\right)$. Order of gum presentation was non-significant $(\mathrm{F}<1)$ as were all interactions involving order of gum presentation. 


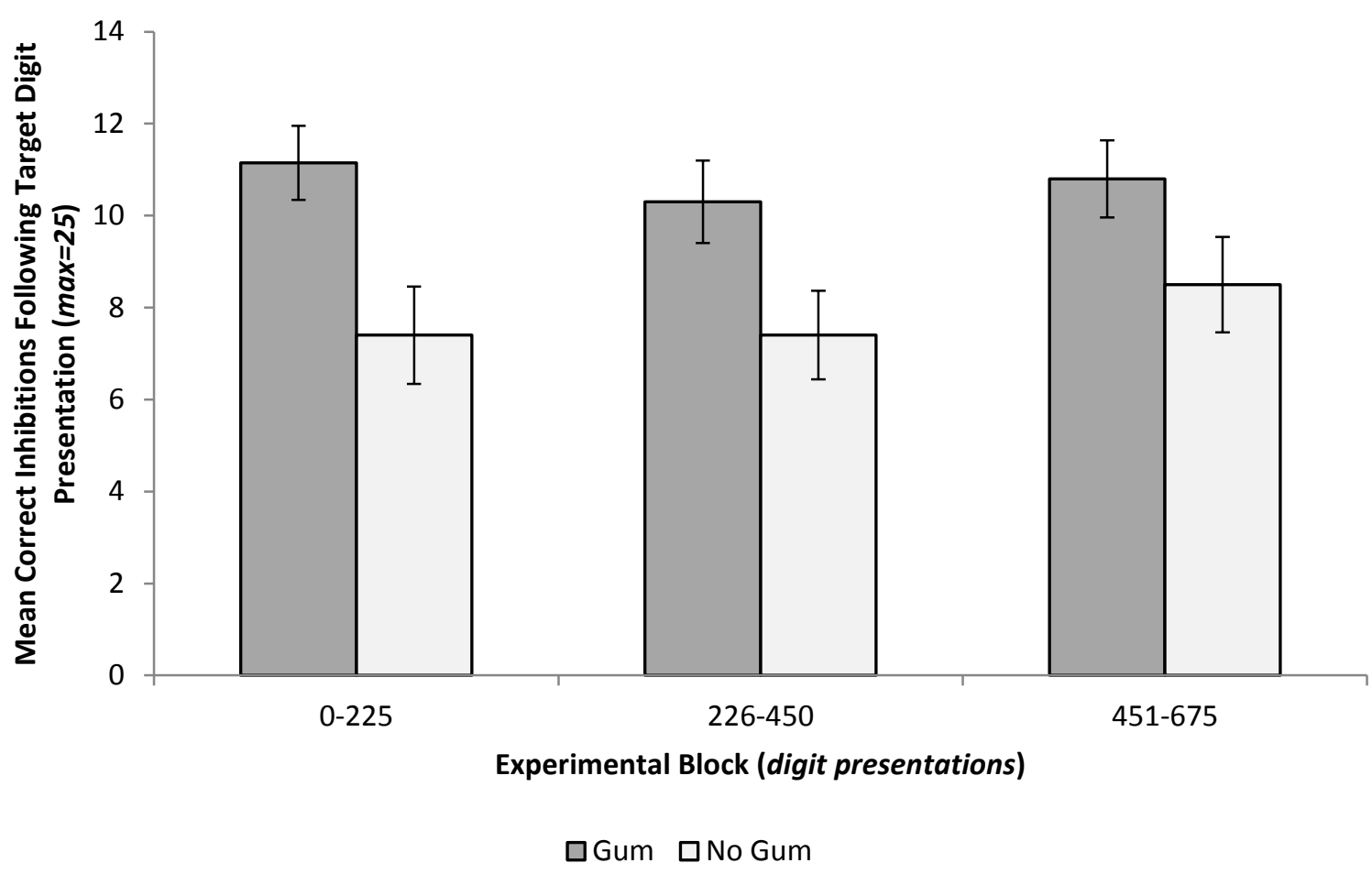

Figure 1: Mean correct response inhibitions following target presentations for the gum and no gum conditions across the three blocks of the SART (digits 0-225, 226-450, and 451-675). Errors bars denote +/- SEM.

The data show a clear performance benefit, in terms of correct target response inhibition, for chewing gum and this benefit is apparent throughout the task. However, it is possible that this effect simply reflects a general behavioural characteristic whilst chewing gum, that is, a reduction in overall response frequency. This possibility was examined by analysing the frequency of correct responses to non-targets. The same $2 \times 2 \times 3$ mixed ANOVA as described for SART scores revealed null effects for gum condition $(F(1,18)=3.30, p=0.09$, partial $\eta^{2}=0.16$ : mean errors for gum and no gum $=164.42$ and 172.85 , respectively), experimental block $\left(F(2,36)=2.37, p=0.11\right.$, partial $\left.\eta^{2}=0.12\right)$ and the chewing gum by experiment stage interaction $\left(F(2,36)=0.29, p=0.75\right.$, partial $\left.\eta^{2}=0.02\right)$. There were no effects or interactions involving the order of gum presentation. It is noteworthy that although the effect of gum condition approached significance, the means indicate a greater response frequency in the gum condition. These data therefore show no support for the possibility that the superior correct target response inhibitions whilst chewing gum reflect a behavioural disinclination to respond to non-target stimuli.

Tucha and Simpson ${ }^{5}$ examined correct reaction times as a measure of the effects of gum on attention. The inhibition of responding required for the SART task does, of course, preclude an assessment of reaction times. However, one can assess the extent to which chewing gum affected response times for the non-targets (as displayed in Figure 2). The same 2x2x3 mixed ANOVA as described above was computed where the dependent variable was the mean 
correct reaction time (ms) for non-targets (both erroneous responses for targets and erroneous response inhibitions for non-targets were omitted). The ANOVA revealed significantly shorter response times for the gum condition, $F(1,18)=19.15$, $p<0.001$, partial $\eta^{2}=0.52$ : mean reaction time for gum and no gum $=259.09 \mathrm{~ms}$ and $297.68 \mathrm{~ms}$, respectively. The main effect of experimental block was non-significant $\left(F(1,18)=2.77, p=0.08\right.$, partial $\left.\eta^{2}=0.13\right)$, as was the block by gum interaction $(F<1)$. There was no main effect of order of gum presentation, nor did presentation order interact with any of the other variables.

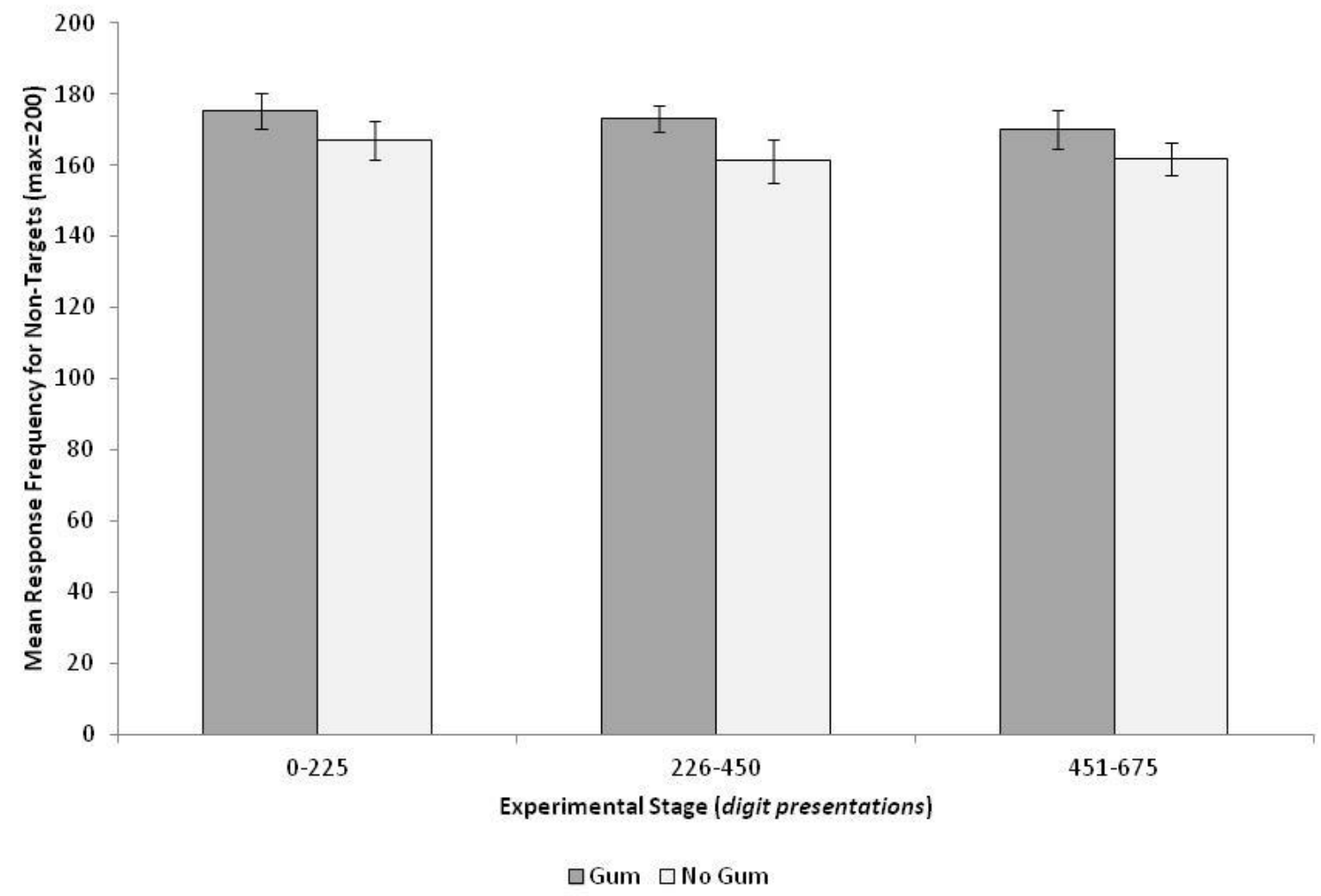

Figure 2: Mean response frequency for non-targets for the gum and no gum conditions across the three stages of the SART (digits 0-225, 226-450, and 451-675). Errors bars denote +/- SEM.

\section{$\underline{\text { Subjective Evaluations of Mood }}$}

For the three measure of mood (alertness, contentedness, and calmness) a 3 -factor $(2 \times 2 \times 2)$ mixed ANOVA was conducted comprising the within-participants factors of gum condition (gum versus non-gum) and pre/post-SART, and the between-participants factor of gum presentation order. The descriptive statistics for these analyses are shown in Table 2 (there were no main effects or interactions involving gum presentation order, so this variable is omitted from Table 2).

Table 2: Mean alertness, contentedness, and calmness scores pre- and post-SART. SEM are reported in parentheses. 


\begin{tabular}{llll}
\hline Alertness & Gum & $51.67(3.10)$ & $62.38(2.65)$ \\
& No Gum & $54.85(2.76)$ & $31.13(4.51)$ \\
Contentedness & Gum & $52.63(3.16)$ & $54.73(2.87)$ \\
& No Gum & $52.73(2.76)$ & $36.72(3.60)$ \\
Calmness & Gum & $51.01(4.40)$ & $65.89(3.57)$ \\
& No Gum & $51.60(4.09)$ & $45.33(4.71)$ \\
\hline
\end{tabular}

Alertness: Table 2 shows the mean self-rated alertness scores for the gum and no-gum conditions pre-and post-SART. The main effects of both gum condition $(F(1,18)=20.23$, $p<0.001$, partial $\eta^{2}=0.53$; means $=57.03$ and 42.99 , for gum and no-gum, respectively) and SART task $\left(F(1,18)=6.13, p=0.02\right.$, partial $\eta^{2}=0.25$; means $=53.26$ and 46.75 , for pre- and post-SART, respectively), were significant. Importantly, the predicted interaction between gum condition and pre/post SART was significant, $F(1,18)=38.15, p<0.001$, partial $\eta^{2}=0.68$. Further analysis (planned comparison Bonferroni-corrected t-tests, $\alpha=0.025$ ), revealed no difference between gum and no-gum conditions pre-SART $(t<1)$ but significantly higher alertness scores for the gum condition post-SART $(t(19)=6.70, p<0.001)$. This indicates that the main effect of SART task was underpinned by the interaction. There were no effects or interaction involving the order of gum conditions.

Contentedness: Table 2 shows the mean self-rated contentedness scores for the gum and no gum conditions pre- and post-SART. The main effects of both gum condition $(F(1,18)=9.98$, $p=0.005$, partial $\eta^{2}=0.36$; means $=53.40$ and 44.72 for the gum and no-gum conditions, respectively), and SART task $\left(F(1,18)=5.85, p=0.03\right.$, partial $\eta^{2}=0.25$; means $=52.68$ and 45.44 , for the gum and no-gum conditions, respectively) were significant. Importantly, the predicted interaction between gum condition and pre/post SART was significant, $F(1,18)=7.32, p=0.01$, partial $\eta^{2}=0.29$. Further analysis (planned comparison Bonferronicorrected t-tests, $\alpha=0.025$ ), revealed no difference between the gum conditions pre-SART $(t<1)$ but a significantly higher contentedness score for the gum condition post-SART $(t(19)=3.51, p=0.002)$. This indicates that the main effect of SART task was underpinned by the interaction. There were no effects or interaction involving the order of gum conditions.

Calmness: Table 2 shows the mean self-rated calmness scores for the gum and no gum conditions pre- and post-SART. The main effect of gum condition was significant $\left(F(1,18)=5.84, p=0.02\right.$, partial $\eta^{2}=0.25$; means $=58.45$ and 48.47 for the gum and no gum conditions, respectively). Although the main effect of SART task was non-significant, $F(1,18)=1.00, p=0.33$, partial $\eta^{2}=0.05$, the predicted interaction between gum condition and pre/post SART was significant, $F(1,18)=4.77, p=0.04$, partial $\eta^{2}=0.21$. Further analysis of the interaction (planned comparison Bonferroni-corrected t-tests, $\alpha=0.025$ ), revealed no 
difference between the gum conditions pre-SART $(t<1)$ but significantly higher calmness scores for the gum condition post-SART $(t(19)=3.47, p=0.003)$. There were no effects or interaction involving the order of gum conditions.

\section{$\underline{\text { Subjective-Behavioural Interrelation }}$}

In order to examine the possibility that changes in subjective alertness underpinned the difference between gum and no-gum SART performance, a single factor (gum versus no gum) repeated measures ANCOVA was computed with change in subjective alertness score as the covariate. The ANCOVA revealed no significant difference between gum and no gum conditions on SART performance, $F(1,18)=2.61, p=0.12$, partial $\eta^{2}=0.13$. This finding suggests that the effect of gum on SART performance was driven, in part, by concomitant changes in subjective alertness score.

\section{Discussion}

For the present study, superior performance on the (SART) was evident whilst chewing gum. This facilitative effect is consistent with a range of other studies examining the effects of gum on sustained attention ${ }^{2,5-7}$. Importantly, the data show that this finding does not reflect an artefact of general task disengagement whilst chewing gum. In fact the data demonstrate a bias towards an increase in responding when chewing gum $(p=0.08)$. Further, the observed improvement in sustained attention performance was not contingent upon performance degradation over time: the facilitative effect of gum on SART performance was uniform across the three experimental blocks. This absence of time-on-task effects, a finding inconsistent with Tucha \& Simpson ${ }^{5}$, suggests that gum-induced facilitative effects on attentional performance are not contingent only upon attentional decline. Indeed, the observation of immediate gum effects on attention contradicts the proposal that chewing gum effects are an analogue of the post-activity cognitive benefits of aerobic exercise ${ }^{15}$. Moreover, no initial impairment in attention was observed for the chewing gum condition. This is inconsistent with Tucha \& Simpson ${ }^{5}$ and, in line with the findings of Allen and Smith ${ }^{6}$, challenges the suggestion that chewing gum interferes with cognition due to the employment of overlapping resources ${ }^{15}$.

Consistent with superior SART performance for the gum condition, correct reaction times for the non-targets were both significantly faster for the gum condition and not contingent upon performance degradation over time. The effect sizes for both these main effects were particularly large (both partial $\eta^{2} \mathrm{~S}>0.45$ ) and it is unclear whether these effects are due to specific demands of the SART paradigm. Indeed, the present study is the first to investigate the effects of chewing gum on such a response inhibition task. Prior gum and attention experiments have used a range of attention tasks and dependent variables, with methodological differences perhaps accounting for the variability in results $2,5,24,25$. Notwithstanding this it is of interest to note that Tucha \& Simpson ${ }^{5}$ did not report a main effect of chewing gum on reaction times in their attention task $\left(\eta^{2}=0.015\right)$. In their study, targets were presented irregularly and relatively infrequently (one target per minute compared to one target every $1150 \mathrm{~ms}$ in the present study). One might speculate, therefore, that gum is 
more effective in facilitating responses to frequent and temporally predictable targets. However, it should be noted that the gum flavour differed across the two studies; spearmint was used in Tucha and Simpson ${ }^{5}$ and menthol gum was employed in the present study. It is possible, therefore, that the menthol flavour in the present study underpinned the strong effect size. The role of gum flavour in the maintenance/elevation of attention clearly merits further examination.

Despite the absence of any time-on-task behavioural decrements in sustained attention, taskinduced mood effects were observed. Both self-rated alertness and contentedness post-SART scores were significantly depressed in the no-gum condition. This suggests a degree of dissociation between the participants' behavioural performance, which remained stable over time, and their perceptions of alertness, which reduced over time. However, chewing gum acted to maintain and increase perceived alertness, maintain contentedness, and increase calmness post-SART, compared to the no-gum condition. The increase in self-rated alertness is consistent with a range of experimental contexts in which chewing gum has resulted in increments in post-task self-rated alertness ${ }^{1,3,4}$. For the present study, chewing gum elevated self-rated alertness following a post-task decline in alertness relative to baseline levels (see also ${ }^{1}$ ). However, this elevation has previously been found in the absence of post-task decrements in perceived alertness ${ }^{2-4}$. Such findings indicate that gum can elevate perceived alertness irrespective of the effects of the task on alertness levels, i.e. a decline in perceived alertness is not a pre-requisite for chewing gum to boost subjective alertness.

The dissociation of time-on-task effects for subjective alertness and attention reported above is consistent with past studies where time-on-task effects have been found with attentional task performance ${ }^{5}$ but not self-rated alertness ${ }^{2}$, and in studies where changes in self-rated alertness have been observed but not changes in attentional task performance ${ }^{16}$. Such dissociation might lead to the conclusion that behavioural attention and perceived alertness are independent constructs. However, whilst these constructs may be, to some extent, differentially sensitive to the chewing of gum, inclusion of alertness shift difference (between gum and no gum) as a co-variable served to abolish the effect of gum on SART performance. This indicates a degree of inter-relation between perceived alertness and attentional task performance, although it should be noted that the correlation between gum/no gum SART task performance difference and gum/no gum alertness change difference was nonsignificant, $r(18)=0.18, p=0.44)$. These variables are not, therefore, interchangeable and gum may, to some extent, act differentially on these constructs.

The precise causal mechanism for these effects (and contributory role of demand characteristics $^{20}$ ) remains unclear. Increases in both cerebral blood flow and activity following the chewing of gum ${ }^{8,9}$ may explain the benefits. However, the lack of carry-over effects reported by Allen and Smith ${ }^{6}$ demonstrates that the effects are not in line with the temporal trajectory of changes in cerebral blood flow post-chewing ${ }^{14}$. Furthermore, the relative immediacy of gum effects on SART performance is inconsistent with the proposal that gum effects are found following a post-chewing delay due to an accentuation of arousal post exercise (i.e. chewing) $)^{15}$. An alternative, or contributory, factor is flavour. Mint is 
associated with increases in attention ${ }^{13}$ and only a small number of studies have considered gum effects in the absence of flavour ${ }^{24,28}$.

The current data contributes to the growing corpus of studies showing that chewing gum can facilitate sustained attention and elevate perceptions of alertness. These studies suggest that chewing gum may provide an inexpensive everyday intervention in aiding attention and alertness. Allen \& Smith $^{6}$ have suggested that such boosts in attention may be acute. However, the extent to which individuals habituate to the benefits of gum following prolonged usage are, to date, unknown. Future studies are required to determine the extent to which chewing gum can operate as a long-term intervention.

\section{References}

${ }^{1}$ Scholey A, Haskell C, Robertson B, Kennedy D, Milne A, Wetherell M. Chewing gum alleviates negative mood and reduces cortisol during acute laboratory psychological stress. Physiol and Behav 2009; 97: 304-312.

${ }^{2}$ Smith A. Effects of chewing gum on cognitive functions, mood and physiology in stressed and non-stressed individuals. Nutr Neurosci 2010; 13(1): 7-16.

${ }^{3}$ Johnson AJ, Jenks RA, Miles C, Albert M, Cox M. Chewing gum Moderates Multi-Task Induced Shifts in Stress, Mood, and Alertness: A Re-Examination. Appetite 2011; 56: 408411.

${ }^{4}$ Sketchley-Kaye K, Jenks RA, Miles C, Johnson AJ. Chewing gum modified state anxiety and alertness under conditions of social stress. Nutr Neurosci 2011; 14(6): 237-242.

${ }^{5}$ Tucha L, Simpson W. The role of time on task performance in modifying effects of gum chewing on attention. Appetite 2011; 56(2): 299-301.

${ }^{6}$ Allen AP, Smith AP. Effects of chewing gum and time on task on alertness and attention. Nutr Neurosci 2012; 15(4): 176-185.

${ }^{7}$ Morgan K, Johnson AJ, Miles C. Chewing gum moderates the vigilance decrement. In preparation.

${ }^{8}$ Sesay M, Tanaka A, Ueno Y, Lecaroz P, De Beaufort DG. Assessment of regional cerebral blood flow by xenon-enhanced computed tomography during mastication in humans. Keio J Med 2000; 49: A125-8. 
${ }^{9}$ Fang M, Li JC, Lu G, Gong XY, Yew DT. A fMRI study of age-related differential cortical patterns during cued motor movements. Brain Topogr 2005; 17(3): 127-137.

${ }^{10}$ Hirano Y, Obata T, Kashikura K, Nonaka H, Tachibana A, Ikehira H, Onozuka M.. Effects of chewing in working memory processing. Neurosci Lett 2008; 436: 189-92.

${ }^{11}$ Stephens R. Tunney R.J. Role of glucose in chewing gum-related facilitation of cognitive function. Appetite 2004; 43: 211-3.

${ }^{12}$ Norrish MI, Dwyer KL. Preliminary investigation of the effect of peppermint oil on an objective measure of daytime sleepiness. Int J Psychophysiol 2005; 55: 291-8.

${ }^{13}$ Warm JS, Dember WN, Parasuraman R. Effects of olfactory stimulation on performance and stress in a visual sustained attention task. J Soc Cosmet Chem 1991; 42: 199-210.

${ }^{14}$ Shinagawa H, Ono T, Honda E, Sasaki T, Taira M, Iriki, A Kuroda T, Ohyama K. Chewing-side preference is involved in differential cortical activation patterns during tongue movement after bilateral gum chewing. A functional magnetic resonance imaging study. $\mathbf{J}$ Dent Res 2004; 83(10): 762-766.

${ }^{15}$ Onyper SV, Carr TL, Fararr JS, Floyd BR. Cognitive advantages of chewing gum. Now you see them, now you don't. Appetite 2011; 57(2): 321-328.

${ }^{16}$ Smith A. Effects of caffeine in chewing gum on mood and attention. Hum Psychopharm Clin 2009a; 24: 239-247.

${ }^{17}$ Johnson AJ, Miles C, Haddrell B, Harrison E, Osborne L, Wilson N, Jenks R. The effect of chewing gum on physiological and self-rated measures of alertness. Physiol and Behav 2012; 105: 815-820.

18 Leproult R, Colecchia EF, Berardi AM, Stickgold R, Kosslyn SM, Van Cauter E. Individual differences in subjective and objective alertness during sleep deprivation are stable and unrelated. Am J Physiol Regul Integr Comp Physiol 2003; 284: R280-290

${ }^{19}$ Allen AP, Smith AP. A review of the evidence that chewing gum affects stress, alertness, and cognition. J Behav Neurosci Res 2011; 9(1): 7-23.

${ }^{20}$ Faro D. Changing the future by reshaping the past: the influence of causal belief on estimates of time to onset. J Consum Res 2010; 37(2): 279-291. 
21 Oken BS, Salinsky MC, Elsas SM. Vigilance, alertness, or sustained attention: physiological basis and measurement. Clin Neurophysiol 2006; 117(9): 1885-1901.

${ }^{22}$ Gray G, Miles C, Wilson N, Jenks R, Cox M, Johnson AJ. The contrasting physiological and subjective effects of chewing gum on social stress. Appetite 2012; 58: 554-558.

${ }^{23}$ Smith A. Effects of chewing gum on mood, learning, memory and performance of an intelligence test. Nutr Neurosci 2009b; 12(2): 81-88.

${ }^{24}$ Tucha O, Mecklinger L, Maier K, Hammerl L, Lange KW. Chewing gum differentially affects aspects of attention in healthy subjects. Appetite 2004; 42(3): 327-329.

${ }^{25}$ Wilkinson L, Scholey A, Wesnes K. Chewing gum selectively improves aspects of memory in healthy volunteers. Appetite 2002; 38: 235-236.

${ }^{26}$ Robertson IH, Manly T, Andrade J, Baddeley BT, Yiend J. 'Oops!': Performance correlates of everyday failure in traumatic brain injured and normal subjects. Neuropsychologia 1997; 35(6): 747-758.

${ }^{27}$ Bond A, Lader M. The use of analogue scales in rating subjective feeling. Br J Med Psychol 1974; 47(3): 211-218.

${ }^{28}$ Johnson AJ, Miles C. Chewing gum and context dependent memory: the independent roles of chewing gum and mint flavour. Br J Psychol 2008; 99: 293-306. 